\title{
SUPPLEMENTARY MATERIAL FOR: Novel synthesis of isoquinolines using isobenzofuran-nitrile Diels-Alder reactions
}

\author{
Binay K. Ghorai, Delu Jiang, and James W. Herndon* \\ Department of Chemistry and Biochemistry, New Mexico State University, MSC 3C, Las \\ Cruces, New Mexico 88003
}

General Procedure 1: Synthesis of Carbene Complexes 4a-c: To a solution of carbene complex 1a-c $(1 \mathrm{mmol})$ in ether $(20 \mathrm{~mL})$ was added $n$-butyllithium $(1 \mathrm{mmol})$ at $-78{ }^{0} \mathrm{C}$. After $0.5 \mathrm{~h}$ this solution was added to a solution of bromoacetonitrile $(10 \mathrm{mmol})$ in $\mathrm{THF}$ $(20 \mathrm{~mL})$ at $0{ }^{\circ} \mathrm{C}$ by means of canula. Mixture was stirred for $2 \mathrm{~h}$ at $0{ }^{\circ} \mathrm{C}$. The solvent was removed in a rotary evaporator. Column chromatography of the crude product over silica gel gave product 4a-c. Complexes 4a-c were quite unstable and were used within a few hours after purification.

Synthesis of Carbene Complex 4a: General Procedure 1 was followed using carbene complex $1 \mathrm{a}(0.400 \mathrm{~g}, 1.6 \mathrm{mmol}), n$-butyllithium $(2.9 \mathrm{~mL}$ of a $1.4 \mathrm{M}$ hexane solution, 4.0 $\mathrm{mmol})$ and bromoacetonitrile $(0.28 \mathrm{~mL}, 4.0 \mathrm{mmol})$. Purification using column chromatography (silica gel, hexane/ ethyl acetate, $3: 1)$ gave $4 \mathbf{a}(0.400 \mathrm{~g}, 46 \%$ yield) contaminated by $5-10 \%$ of bromoacetonitrile. ${ }^{1} \mathrm{H} \mathrm{NMR}\left(\mathrm{CDCl}_{3}\right): \delta 4.90(\mathrm{~s}, 3 \mathrm{H}), 4.50$ (quintet, $1 \mathrm{H}, \mathrm{J}=6.6 \mathrm{~Hz}), 2.65(\mathrm{dd}, 2 \mathrm{H}, \mathrm{J}=17.4,6.6 \mathrm{~Hz}), 2.58(\mathrm{dd}, 2 \mathrm{H}, \mathrm{J}=17.4,6.6$ $\mathrm{Hz}) ;{ }^{13} \mathrm{C} \mathrm{NMR}\left(\mathrm{CDCl}_{3}\right): \delta 353.9,222.6,215.8,116.5,69.0,59.3,19.3$; IR (neat): 2259, $2066,1919,1454 \mathrm{~cm}^{-1}$.

Synthesis of Carbene Complex 4b: General Procedure 1 was followed using carbene complex $1 \mathbf{b}(0.30 \mathrm{~g}, 0.92 \mathrm{mmol}){ }^{1} n$-butyllithium $(0.44 \mathrm{~mL}$ of a $1.6 \mathrm{M}$ hexane solution, $0.70 \mathrm{mmol})$ and bromoacetonitrile $(0.85 \mathrm{~g}, 0.71 \mathrm{mmol})$. Purification using column chromatography (silica gel, hexane/ ethyl acetate, $4: 1)$ gave $\mathbf{4 b}(0.14 \mathrm{~g}, 88 \%$ yield) and starting material $1 \mathbf{b}(0.16 \mathrm{~g}) .{ }^{1} \mathrm{H}$ NMR $\left(\mathrm{CDCl}_{3}\right): \delta 4.70(\mathrm{~s}, 3 \mathrm{H}), 4.45(\mathrm{~m}, 1 \mathrm{H}), 2.45-2.78$ $(\mathrm{m}, 4 \mathrm{H}), 1.60-1.78(\mathrm{~m}, 6 \mathrm{H}), 1.25-1.55(\mathrm{~m}, 12 \mathrm{H}), 0.97$ (br t, $9 \mathrm{H}, \mathrm{J}=6.8 \mathrm{~Hz}) ;{ }^{13} \mathrm{C} \mathrm{NMR}$ $\left(\mathrm{CDCl}_{3}\right): \delta 338.6(\mathrm{~d}), 222.2,221.9,116.8,64.6,58.3,27.8(\mathrm{~d}), 25.4,24.3(\mathrm{~d}), 18.8,13.6$; IR (neat): $2235,2008,1883 \mathrm{~cm}^{-1}$.

Synthesis of Carbene Complex 4c: General Procedure 1 was followed using carbene complex 1c $(0.52 \mathrm{~g}, 1.78 \mathrm{mmol}), n$-butyllithium $(1.1 \mathrm{~mL}$ of a $1.6 \mathrm{M}$ hexane solution, 1.76 $\mathrm{mmol})$ and bromoacetonitrile $(2.14 \mathrm{~g}, 17.8 \mathrm{mmol})$. Purification using column chromatography ( silica gel, hexane/ ethyl acetate, 9:1) gave $4 \mathrm{c}(0.310 \mathrm{~g}, 52 \%) .{ }^{1} \mathrm{H}$ NMR $\left(\mathrm{CDCl}_{3}\right): \delta 4.849(\mathrm{~s}, 3 \mathrm{H}), 4.22(\mathrm{~m}, 1 \mathrm{H}), 2.39(\mathrm{t}, 1 \mathrm{H}, \mathrm{J}=6.5 \mathrm{~Hz}), 1.2-1.8(\mathrm{~m}, 5 \mathrm{H}), 0.95$ (t, $3 \mathrm{H}, \mathrm{J}=6.5 \mathrm{~Hz})$; IR(neat): 2240, 2063, $1919 \mathrm{~cm}^{-1}$.

\footnotetext{
${ }^{1}$ Xu, Yao Chang; Wulff, William D. J. Org. Chem. 1987, 52, 3263-3275.
} 
General Procedure 2: Synthesis of Carbene Complexes 4d and 6a: ${ }^{2}$ 1) Preparation of Dipotassium Pentacarbonylchromate: Graphite (64 eq) was heated at $150{ }^{\circ} \mathrm{C}-160{ }^{\circ} \mathrm{C}$ while being stirred under argon for $2 \mathrm{~h}$. The graphite was cooled to room temperature and potassium ( 8 eq) was added as tiny pieces under a positive flow of argon. The mixture was heated to $160{ }^{\circ} \mathrm{C}$ with stirring for $1 \mathrm{~h}$. THF was added to the bronze colored material and cooled to $-78{ }^{\circ} \mathrm{C}$. Chromium hexacarbonyl (3.5 eq) was then added under a positive flow of argon. The reaction mixture was stirred for $30 \mathrm{~min}$ at $-78{ }^{\circ} \mathrm{C}$ and placed in an ice bath until a thick slurry of product was formed. This mixture was directly used in the next step. 2) Preparation of tetramethylammonium salt of the chromium carbene complex. To the solution of dipotassium pentacarbonylchromate in THF at $-78{ }^{\circ} \mathrm{C}$ obtained from step1, the acid chloride (1 eq) in THF was added dropwise. The resulting mixture was stirred at $-78{ }^{\circ} \mathrm{C}$ for $15 \mathrm{~min}$, at $0{ }^{\circ} \mathrm{C}$ for $1.5 \mathrm{~h}$, and at room temperature for 1 $\mathrm{h}$. The solvent was removed under reduced pressure. Cold water was added to the residue and filtered through Celite. To the filtrate was added a saturated aqueous solution of tetramethylammonium bromide, leading to immediate precipitation of the ammonium salt. The precipitate was removed by extracting the aqueous layer with dichloromethane. The organic layer was then dried over anhydrous sodium sulfate and the solvent was removed on a rotary evaporator to give a pale yellow solid. 3) Alkylation of tetramethylammonium acylate salt. To a solution of the ammonium salt (1 eq) in dichloromethane obtained in step 2 , methyl triflate $(1.2 \mathrm{eq})$ was added at $0{ }^{\circ} \mathrm{C}$. The reaction mixture was then stirred at $0{ }^{\circ} \mathrm{C}$ for $0.5 \mathrm{~h}$ and at room temperature for $1 \mathrm{~h}$. The mixture was filtered through Celite, washed with saturated aqueous sodium bicarbonate solution, water, brine, and dried over sodium sulfate. The solvent was removed on a rotary evaporator to yield the carbene complex. Purification was done by flash column chromatography using hexane-ethyl acetate (4:1) as the eluting solvent. Both of these complexes were highly unstable and used within a few hours after purification.

Synthesis of Carbene Complex 4d: General Procedure 2 was followed using dipotassium pentacarbobylchromate (prepared from graphite [2.34 g, $195 \mathrm{mmol}$ ), potassium metal (0.95 g, $24.3 \mathrm{mmol})$, and chromium hexacarbonyl (2.42 g, $11.0 \mathrm{mmol})$ ], cis 2-cyanocyclohexanecarbonyl chloride $(0.55 \mathrm{~g}, 3.2 \mathrm{mmol}),{ }^{3}$ and methyl triflate $(0.63 \mathrm{~g}$, $3.84 \mathrm{mmol})$. The crude product was purified by flash chromatography to give $\mathbf{4 d}$, dark yellow solid (0.670 g, 61\%). ${ }^{1} \mathrm{H}$ NMR $\left(\mathrm{CDCl}_{3}\right)$ : $\delta 4.90(\mathrm{~s}, 3 \mathrm{H}), 3.96(\mathrm{~m}, 1 \mathrm{H}), 3.08(\mathrm{~m}, 1$ $\mathrm{H}), 1.58-2.20(\mathrm{~m}, 6 \mathrm{H}), 1.25-2.20(\mathrm{~m}, 6 \mathrm{H}), 1.25-1.58(\mathrm{~m}, 2 \mathrm{H}) ;{ }^{13} \mathrm{C} \mathrm{NMR}\left(\mathrm{CDCl}_{3}\right): \delta$ $358.3,215.6,214.2,130.8,69.6,68.2,30.6,29.6,25.6,24.8,22.0$; IR(neat): 2264, 2063, $1919 \mathrm{~cm}^{-1}$.

\footnotetext{
${ }^{2}$ This is a variant of a literature procedure: Schwindt, M..A.; Lejon, T.; Hegedus, L.S. Organometallics 1990, 9, 2814-2819. This procedure is very reliable if one is careful to cut the potassium into small pieces and if the tetramethylammonium acylate salt is prepared prior to the alkylation step. Otherwise we noted wide variances in the yields between different experimental runs.

${ }^{3}$ Prepared from cis 1,2-cyclohexanedicarboxylic anhydride via amide formation (treatment with aqueous ammonia), followed by amide dehydration (acetic anhydride), followed by acid chloride formation (oxalyl chloride)
} 
Synthesis of Carbene Complex 6a: General Procedure 2 was followed using dipotassium pentacarbobylchromate [prepared from graphite $(2.34 \mathrm{~g}, 195 \mathrm{mmol})$, potassium metal $(0.95 \mathrm{~g}, 24.3 \mathrm{mmol})$ and chromium hexacarbonyl $(2.42 \mathrm{~g} 11.0 \mathrm{mmol})]$, acid chloride 5a $(0.53 \mathrm{~g}, 3.2 \mathrm{mmol})$, and methyl triflate $(0.63 \mathrm{~g}, 3.84 \mathrm{mmol})$. The crude product was purified by passage through a short silica gel column to give $6 \mathbf{6}(0.378 \mathrm{~g}$, $35 \%$ yield) and was immediately used for next step as it is an unstable compound.

${ }^{1} \mathrm{H}$ NMR $\left(\mathrm{CDCl}_{3}\right)$ (poorly resolved): $\delta$ 7.52-7.80 (m, $\left.2 \mathrm{H}\right), 7.30-7.50(\mathrm{~m}, 2 \mathrm{H}), 4.95$ (s, 3 H); IR: 2068, $1930 \mathrm{~cm}^{-1}$.

General Procedure 3: Synthesis of Carbene Complexes 4e and 6b: To the solution of dipotassium pentacarbonylchromate (prepared using general procedure 2, step 1) in THF at $-78{ }^{\circ} \mathrm{C}$, the amide $(1 \mathrm{eq})$ in THF was added dropwise. The resultant mixture was stirred at $-78{ }^{\circ} \mathrm{C}$ for $0.5 \mathrm{~h}$, warmed to $0{ }^{\circ} \mathrm{C}$ for $1 \mathrm{~h}$, and cooled to $-78{ }^{\circ} \mathrm{C}$.

Chlorotrimethylsilane ( $3 \mathrm{eq}$ ) was then added. After $0.5 \mathrm{~h}$, the mixture was warmed to room temperature. Neutral $\mathrm{Al}_{2} \mathrm{O}_{3}(2.5 \mathrm{~g} / 1 \mathrm{mmol}$ of amide) was added to absorb the product, and the solvent was removed under reduced pressure. The resulting dry powder was chromatographed on a silica gel column with hexane-dichloromethane (1:1) as eluent.

Synthesis of Carbene Complex 4e: General Procedure 3 was followed using dipotassium pentacarbobylchromate [prepared from graphite $(1.78 \mathrm{~g}, 148 \mathrm{mmol})$, potassium metal $(0.72 \mathrm{~g}, 18.5 \mathrm{mmol})$ and chromium hexacarbonyl $(2.42 \mathrm{~g} 8.4 \mathrm{mmol})]$, cis N,N-dimethyl-2-cyanocyclohexanecarboxamide (0.82 g, $4.55 \mathrm{mmol}){ }^{4}$ and chlorotrimethylsilane $(1.50 \mathrm{~g}, 14.0 \mathrm{mmol})$. The crude product was purified by flash chromatography to give $4 \mathrm{e}(0.650 \mathrm{~g}, 40 \%) .{ }^{1} \mathrm{H} \mathrm{NMR}\left(\mathrm{CDCl}_{3}\right): \delta 3.98(\mathrm{~s}, 3 \mathrm{H}), 3.93$ (br s, $1 \mathrm{H}), 3.55(\mathrm{~s}, 3 \mathrm{H}), 3.29(\mathrm{bs}, 1 \mathrm{H}), 1.3-2.3(\mathrm{~m}, 8 \mathrm{H}) ;{ }^{13} \mathrm{C} \mathrm{NMR}\left(\mathrm{CDCl}_{3}\right): \delta 280.0,217.2$, 213.8, 131.1, 62.0, 56.1, 46.7, 31.7, 31.3, 28.2, 26.2, 22.3; IR (neat): 2053, $1903 \mathrm{~cm}^{-1}$.

Synthesis of Carbene Complex 6b: General Procedure 3 was followed using dipotassium pentacarbobylchromate [prepared from graphite $(2.34 \mathrm{~g}, 195 \mathrm{mmol})$, potassium metal $(0.95 \mathrm{~g}, 24.3 \mathrm{mmol})$ and chromiumhexacarbonyl $(2.42 \mathrm{~g} 11.0 \mathrm{mmol})]$, amide $5 \mathbf{b}^{5}$ (1.04 g, $\left.6.0 \mathrm{mmol}\right)$ and chlorotrimethylsilane $(1.95 \mathrm{~g}, 18.0 \mathrm{mmol})$. The crude product was purified by flash chromatography to give $6 \mathbf{b}(1.35 \mathrm{~g}, 64 \%)$. ${ }^{1} \mathrm{H}$ NMR $\left(\mathrm{CDCl}_{3}\right): \delta 7.55-7.78(\mathrm{~m}, 2 \mathrm{H}), 7.34(\mathrm{~d}, 1 \mathrm{H}, \mathrm{J}=8 \mathrm{~Hz}), 6.95(\mathrm{~d}, 1 \mathrm{H}, \mathrm{J}=8 \mathrm{~Hz}), 4.13(\mathrm{~s}, 3$ $\mathrm{H}), 3.19(\mathrm{~s}, 3 \mathrm{H}) ;{ }^{13} \mathrm{C} \mathrm{NMR}\left(\mathrm{CDCl}_{3}\right): \delta 272.3,216.1,213.8,154.7,134.0,133.0,126.7$, 121.4, 100.6, 51.4, 46.4; IR(neat): $2057,1918 \mathrm{~cm}^{-1}$.

\footnotetext{
${ }^{4}$ Prepared from reaction of the acid chloride (footnote 3 ) with aqueous dimethylamine.

${ }^{5}$ Armarego, W.L.F.; Sharma, S.C. J. Chem. Soc. C 1970, 1600-1606.
} 
General Procedure 4:Coupling of $\beta$-cyanocarbene Complexes $(4,6)$ with $o$-alkynyl benzaldehyde/ $o$-alkynyl acetophenones (2): To a refluxing solution of 1.0 eq of $o$ alkynyl benzaldehyde/ $o$-alkynyl acetophenone (2) in a minimum volume of dioxane under argon was added dropwise a $0.05 \mathrm{M}$ solution of carbene complex $(1.1 \mathrm{eq})$ in dioxane over a $2 \mathrm{~h}$ period. After the addition was complete, the mixture was allowed to reflux for a period of 16-24 $\mathrm{h}$. The reaction mixture was cooled to room temperature and concentrated on a rotary evaporator. Ethyl acetate $(25 \mathrm{~mL})$ was added and the residue was filtered through Celite. The solvent was removed on a rotary evaporator and the crude products were purified by flash column chromatography.

\section{Coupling of Carbene Complex 4a with 2-hex-1-ynylbenzaldehyde (2a) (Entry A):} General Procedure 4 was followed using carbene complex $4 \mathbf{a}(100 \mathrm{mg}, 0.30 \mathrm{mmol})$ and 2-hex-1-ynylbenzaldehyde (2a) $(60 \mathrm{mg}, 0.32 \mathrm{mmol})$. The crude product was purified using flash chromatography on silica gel with hexane-ethyl acetate (1:1) as eluent to yield products $7 \mathbf{a}(36 \mathrm{mg}, 40 \%), 8(29 \mathrm{mg}, 36 \%)$, and a compound tentatively identified as enol ether 9 (5 mg, 13\%, yield varies widely between experimental runs). Compound $7 a$ : ${ }^{1} \mathrm{H}$ NMR $\left(\mathrm{CDCl}_{3}\right): \delta 8.99(\mathrm{~s}, 1 \mathrm{H}), 8.09(\mathrm{~d}, 1 \mathrm{H}, \mathrm{J}=8.4 \mathrm{~Hz}), 7.94(\mathrm{~d}, 1 \mathrm{H}, \mathrm{J}=8.4 \mathrm{~Hz}), 7.67$ (t, $1 \mathrm{H}, \mathrm{J}=7.5 \mathrm{~Hz}), 7.55(\mathrm{t}, 1 \mathrm{H}, \mathrm{J}=7.5 \mathrm{~Hz}), 3.84(\mathrm{~s}, 3 \mathrm{H}), 3.45(\mathrm{~m}, 1 \mathrm{H}), 3.00-3.30(\mathrm{~m}, 2$ $\mathrm{H}), 2.65-3.00(\mathrm{~m}, 2 \mathrm{H}), 2.18-2.5(\mathrm{~m}, 2 \mathrm{H}), 1.10-1.50(\mathrm{~m}, 4 \mathrm{H}), 0.80(\mathrm{t}, 3 \mathrm{H}, \mathrm{J}=7 \mathrm{~Hz}) ;{ }^{13} \mathrm{C}$ NMR $\left(\mathrm{CDCl}_{3}\right): \delta 156.8,148.4,144.6,133.0,130.5,128.8,128.3,126.7,124.5,118.4$, 118.1, 58.1, 36.6, 31.0, 30.4, 28.2, 22.5, 18.8, 13.7; IR (neat): 2239, 1615, $1551 \mathrm{~cm}^{-1}$; MS: (m/e) $307\left(\mathrm{M}^{+}+1,13\right), 306\left(\mathrm{M}^{+}, 55\right), 291\left(\mathrm{M}^{+}-\mathrm{CH}_{3}, 10\right), 266$ (45), 210 (100), 180 (21), 167 (24). HRMS: Calcd. For $\mathrm{C}_{20} \mathrm{H}_{22} \mathrm{~N}_{2} \mathrm{O}$ is 306.17322, found 306.17373.

Compound 8: ${ }^{1} \mathrm{H}$ NMR $\left(\mathrm{CDCl}_{3}\right): \delta 9.10($ br s, $1 \mathrm{H}), 8.69(\mathrm{~d}, 1 \mathrm{H}, \mathrm{J}=8.4 \mathrm{~Hz}), 8.12(\mathrm{~d}, 1$ $\mathrm{H}, \mathrm{J}=9.0 \mathrm{~Hz}), 8.04(\mathrm{~d}, 1 \mathrm{H}, \mathrm{J}=7.7 \mathrm{~Hz}), 7.84(\mathrm{t}, 1 \mathrm{H}, \mathrm{J}=7.3 \mathrm{~Hz}), 7.71(\mathrm{t}, 1 \mathrm{H}, \mathrm{J}=7.3$ $\mathrm{Hz}), 7.44(\mathrm{~d}, 1 \mathrm{H}, \mathrm{J}=9.0 \mathrm{~Hz}), 4.01(\mathrm{~s}, 3 \mathrm{H}), 3.35$ (t, $2 \mathrm{H}, \mathrm{J}=8.0 \mathrm{~Hz}), 1.75-1.98(\mathrm{~m}, 2 \mathrm{H})$, $1.50-1.75(\mathrm{~m}, 2 \mathrm{H}), 1.09$ (t, $3 \mathrm{H}, \mathrm{J}=7.5 \mathrm{~Hz}) ;{ }^{13} \mathrm{C} \mathrm{NMR}\left(\mathrm{CDCl}_{3}\right): \delta 157.1,150.8,139.8$, 133.2, 130.3, 129.2, 128.8, 127.3, 127.0, 126.6, 113.3, 56.4, 30.8, 28.2, 23.2, 13.9; IR (neat): 2957, 2871, 1616, $1495 \mathrm{~cm}^{-1}$; MS: (m/e) $267\left(\mathrm{M}^{+}+2,6\right), 266\left(\mathrm{M}^{+}+1,26\right), 265\left(\mathrm{M}^{+}\right.$, 54), 222 (100), 206 (5), 191 (7), 179 (29), 152 (7); HRMS: Calcd. For $\mathrm{C}_{18} \mathrm{H}_{19} \mathrm{NO}$ is 265.146664, found 265.145879. Compound 9: ${ }^{1} \mathrm{H} \mathrm{NMR}\left(\mathrm{CDCl}_{3}\right): \delta 6.40$ (br s, $\left.1 \mathrm{H}\right)$; 3.73 (s, $3 \mathrm{H}), 3.28$ (s, $2 \mathrm{H}), 3.14$ (s, $2 \mathrm{H})$; IR (neat): 2248 (m), 1652 (s) $\mathrm{cm}^{-1} .6$

Coupling of Carbene Complex 4b with 2-hex-1-ynyl-benzaldehyde (2a) (Entry B): General Procedure 4 was followed using carbene complex $4 \mathbf{b}(0.165 \mathrm{~g}, 0.33 \mathrm{mmol})$ and 2-hex-1-ynylbenzaldehyde (2a) $(0.056 \mathrm{~g}, 0.30 \mathrm{mmol})$. The crude product was purified using flash chromatography on silica gel with hexane-ethyl acetate $(1: 1)$ as eluent to yield products $7 \mathbf{a}(0.044 \mathrm{~g}, 48 \%)$ and $\mathbf{8}(0.015 \mathrm{~g}, 19 \%)$.

Coupling of Carbene Complexe 4c with 2-hex-1-ynyl-benzaldehyde (2a) (Entry C): General Procedure 4 was followed using carbene complex $4 \mathbf{c}(0.150 \mathrm{~g}, 0.45 \mathrm{mmol})$ and

\footnotetext{
${ }^{6}$ This compound is known however the spectral data is inaccessible: Chem. Abstr. 1976, 86, 189240.
} 
2-hex-1-ynyl-benzaldehyde (2a) (0.075 g, $0.40 \mathrm{mmol})$; in this experiment triphenylphosphine $(0.118 \mathrm{~g}, 0.45 \mathrm{mmol})$ was added and toluene was used as the solvent (in place of dioxane). The crude product was purified using flash chromatography on silica gel with hexane-ethyl acetate (4:1) as eluent to yield product $12 \mathrm{c}(0.065 \mathrm{~g}, 53 \%)$. ${ }^{1} \mathrm{H}$ NMR $\left(\mathrm{CDCl}_{3}\right): \delta 9.15(\mathrm{~s}, 1 \mathrm{H}), 8.71(\mathrm{~d}, 1 \mathrm{H}, \mathrm{J}=8 \mathrm{~Hz}), 8.04(\mathrm{~d}, 1 \mathrm{H}, \mathrm{J}=8.0 \mathrm{~Hz}), 7.96$ (s, $1 \mathrm{H}), 7.83(\mathrm{t}, 1 \mathrm{H}, \mathrm{J}=7.7 \mathrm{~Hz}), 7.68(\mathrm{t}, 1 \mathrm{H}, \mathrm{J}=7.7 \mathrm{~Hz}), 3.85(\mathrm{~s}, 3 \mathrm{H}), 3.43(\mathrm{t}, 2 \mathrm{H}, \mathrm{J}=$ $8.0 \mathrm{~Hz}), 2.87(\mathrm{t}, 2 \mathrm{H}, \mathrm{J}=7.7 \mathrm{~Hz}), 1.70-1.93(\mathrm{~m}, 4 \mathrm{H}), 1.52-1.70(\mathrm{~m}, 2 \mathrm{H}), 1.05(\mathrm{t}, 3 \mathrm{H}, \mathrm{J}=$ $7.3 \mathrm{~Hz}), 1.03(\mathrm{t}, 3 \mathrm{H}, \mathrm{J}=7.3 \mathrm{~Hz}) ;{ }^{13} \mathrm{C} \mathrm{NMR}\left(\mathrm{CDCl}_{3}\right): \delta 156.8,152.4,142.8,137.3,133.3$, $132.4,130.3,129.5,129.3,127.3,126.5,126.0,122.4,61.6,32.4,31.8,28.7,23.2(2 \mathrm{C})$, 14.2, 13.9; IR (neat): 2958, 2871, 1591, $1446 \mathrm{~cm}^{-1}$; MS: (m/e) $309\left(\mathrm{M}^{+}+2,3\right), 308\left(\mathrm{M}^{+}+1\right.$, 20), $307\left(\mathrm{M}^{+}, 59\right), 292\left(\mathrm{M}^{+}-\mathrm{CH}_{3}, 4\right), 264$ (100), 234 (16), 222 (9), 204 (9), 192 (13), 165

(5); HRMS: Calcd. For $\mathrm{C}_{21} \mathrm{H}_{25} \mathrm{NO}$ is 307.193615 , found 307.194478

Coupling of Carbene Complex 4c with 2-ethynylbenzaldehyde (7d) (Entry D): General Procedure 4 was followed using carbene complex $4 \mathbf{c}(0.345 \mathrm{~g}, 1.0 \mathrm{mmol})$ and 2ethynylbenzaldehyde (7d) $(0.117 \mathrm{~g}, 0.90 \mathrm{mmol})$. The crude product was purified using flash chromatography on silica gel with hexane-ethyl acetate (4:1) as eluent to yield product 7d $(0.050 \mathrm{~g}, 21 \%) .{ }^{1} \mathrm{H}$ NMR $\left(\mathrm{CDCl}_{3}\right): \delta 8.97(\mathrm{~s}, 1 \mathrm{H}), 8.01(\mathrm{~d}, 1 \mathrm{H}, \mathrm{J}=8.0 \mathrm{~Hz})$, $7.92(\mathrm{~d}, 1 \mathrm{H}, \mathrm{J}=8.0 \mathrm{~Hz}), 7.68(\mathrm{t}, 1 \mathrm{H}, \mathrm{J}=7.5 \mathrm{~Hz}), 7.53(\mathrm{t}, 1 \mathrm{H}, \mathrm{J}=7.5 \mathrm{~Hz}), 6.08(\mathrm{~s}, 1 \mathrm{H})$, $3.57(\mathrm{~s}, 3 \mathrm{H}), 3.45(\mathrm{br} \mathrm{s}, 1 \mathrm{H}$, line width at half height $=15 \mathrm{~Hz}), 2.84(\mathrm{~m}, 1 \mathrm{H}$, line width at half height $=12 \mathrm{~Hz}), 2.28(\mathrm{~m}, 1 \mathrm{H}), 1.35-1.90(\mathrm{~m}, 7 \mathrm{H}) ;{ }^{13} \mathrm{C} \mathrm{NMR}\left(\mathrm{CDCl}_{3}\right): \delta 164.8$, 146.1, 131.8, 130.3, 128.4, 127.3, 126.4, 124.5, 122.0, 89.1, 55.3, 41.8, 39.4, 26.4, 25.2, 24.1, 23.9; IR (neat): 2933, $1634 \mathrm{~cm}^{-1}$; MS: (m/e) $266\left(\mathrm{M}^{+}+1,18\right), 265\left(\mathrm{M}^{+}, 99\right), 264$ (27), $263\left(\mathrm{M}^{+}-\mathrm{H}_{2}, 100\right), 248$ (40), 236 (27), 234 (27), 222 (35), 210 (99), 192 (36), 180 (16), 166 (29); HRMS: Calcd. For $\mathrm{C}_{18} \mathrm{H}_{19} \mathrm{NO}$ is 265.146664 , found 265.145504. The cis stereochemistry was assigned based on the relatively narrow line widths for the ring junction protons at $\delta 3.45$ and $\delta 2.84$. In the trans isomer, these protons would be axial and would couple to two other axial protons, and should feature much wider and discernable patterns.

\section{Coupling of Carbene Complex 4e with 2-ethynylbenzaldehyde (2d) (Entry E):}

General Procedure 4 was followed using carbene complex 4 e $(0.355 \mathrm{~g}, 1.0 \mathrm{mmol})$ and 2ethynylbenzaldehyde (2d) $(0.116 \mathrm{~g}, 0.89 \mathrm{mmol})$; in this experiment triphenylphosphine $(0.262 \mathrm{~g}, 1.0 \mathrm{mmol})$ and toluene was used as the solvent (in place of dioxane). The crude product was purified using flash chromatography on silica gel with hexane-ethyl acetate $(1: 1)$ as eluent to yield product 11e $(0.095 \mathrm{~g}, 42 \%) .{ }^{1} \mathrm{H} \mathrm{NMR}\left(\mathrm{CDCl}_{3}\right): \delta 9.16(\mathrm{~s}, 1 \mathrm{H})$, $7.10(\mathrm{~d}, 1 \mathrm{H}, \mathrm{J}=7.7 \mathrm{~Hz}), 7.70-7.85(\mathrm{~m}, 2 \mathrm{H}), 7.61(\mathrm{~m}, 1 \mathrm{H}), 4.02(\mathrm{~d}, 1 \mathrm{H}, \mathrm{J}=21.8 \mathrm{~Hz})$, $3.76(\mathrm{~d}, 1 \mathrm{H}, \mathrm{J}=21.8 \mathrm{~Hz}$ ), 3.49(ddd, $1 \mathrm{H}, \mathrm{J}=10.7,5.0,3.8 \mathrm{~Hz}$ ), 3.06 (br s, $1 \mathrm{H}$, line width at half height $=10 \mathrm{~Hz}), 2.44(\mathrm{~m}, 1 \mathrm{H}), 2.07(\mathrm{~m}, 1 \mathrm{H}), 1.79(\mathrm{~m}, 1 \mathrm{H}), 1.40-1.70(\mathrm{~m}, 4$ $\mathrm{H}), 1.25(\mathrm{~m}, 1 \mathrm{H}) ;{ }^{13} \mathrm{C} \mathrm{NMR}\left(\mathrm{CDCl}_{3}\right): \delta 209.4,152.6,151.4,134.7,131.0,128.5,127.1$, 126.8, 121.8, 120.4, 48.1 45.8, 38.7, 31.8, 26.0, 24.4, 22.0; IR (neat): 2933, 1713, 1623 $\mathrm{cm}^{-1}$; MS: (m/e) $252\left(\mathrm{M}^{+}+1,23\right), 251\left(\mathrm{M}^{+}, 100\right), 234$ (5), 222 (38), 208 (15), 196 (97), 180 (49), 167 (41), 152 (15), 115 (19); HRMS: Calcd. For $\mathrm{C}_{17} \mathrm{H}_{17} \mathrm{NO}$ is 251.131014, found 251.130288. The cis stereochemistry was assigned based on the appearance the 
ring junction protons at $\delta 3.49(\mathrm{ddd}, \mathrm{J}=10.7,5.0,3.8 \mathrm{~Hz})$, and $\delta 3.06(\mathrm{br} \mathrm{s}$, line width at half height $=10 \mathrm{~Hz}$ ). In the trans isomer, these protons would be axial and would couple to two other axial protons, and should feature much wider and discernable patterns. The pattern at $\delta 3.49$ is consistent with an axial proton coupled to one other axial proton and two equatorial protons, as expected in the cis isomer. The pattern at $\delta 3.06$ is consistent with contribution from multiple small couplings, as expected for an equatorial proton.

\section{Coupling of Carbene Complex 4d with 1-(2-hex-1-ynyl-phenyl)-ethanone (2b)} (Entry F): General Procedure 4 was followed using carbene complex $4 \mathbf{d}(0.10 \mathrm{~g}, 0.29$ mmol) and 1-(2-hex-1-ynyl-phenyl)-ethanone (2b) ( $0.052 \mathrm{~g}, 0.26 \mathrm{mmol})$. The crude product was purified using flash chromatography over silica gel with hexane-ethyl acetate (1:1) as eluent to yield product $7 \mathbf{f}(0.035 \mathrm{~g}, 40 \%) .{ }^{1} \mathrm{H} N M R\left(\mathrm{CDCl}_{3}\right): \delta 8.07(\mathrm{~d}, 2$ $\mathrm{H}, \mathrm{J}=8.0 \mathrm{~Hz}$ ), 7.40-7.60 (m, $2 \mathrm{H}), 3.75$ (s, $3 \mathrm{H}), 3.20$ (m, $1 \mathrm{H}), 2.93$ (s, $3 \mathrm{H}), 2.55-2.92$ $(\mathrm{m}, 3 \mathrm{H}), 1.10-1.60(\mathrm{~m}, 12 \mathrm{H}), 0.77(\mathrm{t}, 3 \mathrm{H}, \mathrm{J}=7.0 \mathrm{~Hz}) ;{ }^{13} \mathrm{C} \mathrm{NMR}\left(\mathrm{CDCl}_{3}\right): \delta 159.4$, 154.3, 149.6, 133.1, 128.6, 128.1, 126.3, 125.9, 125.2, 125.1, 116.5, 57.6, 41.8, 36.4, 31.0, 28.2, 26.3, 25.8, 24.8, 22.8, 22.7, 22.3, 13.8; IR(neat): 2930, $1627 \mathrm{~cm}^{-1}$; MS: (m/e) $337\left(\mathrm{M}^{+}+2,2\right), 336\left(\mathrm{M}^{+}+1,26\right), 335\left(\mathrm{M}^{+}, 100\right), 320\left(\mathrm{M}^{+}-\mathrm{Me}, 31\right), 306\left(\mathrm{M}^{+}-\mathrm{Et}, 9\right), 292$ (35), 280 (22), 262 (10), 224 (10), 145 (15); HRMS: Calcd. For $\mathrm{C}_{23} \mathrm{H}_{29} \mathrm{NO}$ is 335.224.915, found 335.225687 .

\section{Coupling of Carbene Complex 6b with 2-hex-1-ynyl-benzaldehyde (2a) (Entry G):} General Procedure 4 was followed using carbene complex 6a $(0.385 \mathrm{~g}, 1.10 \mathrm{mmol})$ and 2-hex-1-ynyl-benzaldehyde (2a) $(0.186 \mathrm{~g}, 1.0 \mathrm{mmol})$. The crude product was purified using flash chromatography on silica gel with hexane-ethyl acetate (9:1) as eluent to yield product 13g $(0.130 \mathrm{~g}, 40 \%)$. ${ }^{1} \mathrm{H}$ NMR $\left(\mathrm{CDCl}_{3}\right): \delta 9.61$ (overlapping s and $\mathrm{d}, \mathrm{J}=8.0 \mathrm{~Hz}$ ), $8.84(\mathrm{~d}, 1 \mathrm{H}, \mathrm{J}=8.5 \mathrm{~Hz}), 8.21(\mathrm{t}, 2 \mathrm{H}, \mathrm{J}=7.7 \mathrm{~Hz}), 8.00(\mathrm{t}, 1 \mathrm{H}, \mathrm{J}=7.4 \mathrm{~Hz}), 7.82(\mathrm{~m}, 3 \mathrm{H})$, $4.00(\mathrm{~s}, 3 \mathrm{H}), 3.51(\mathrm{t}, 2 \mathrm{H}, \mathrm{J}=8.1), 1.88(\mathrm{~m}, 2 \mathrm{H}), 1.62$ (sextet, $2 \mathrm{H}, \mathrm{J}=7.3 \mathrm{~Hz}), 1.03(\mathrm{t}, 3$ $\mathrm{H}, \mathrm{J}=7.3 \mathrm{~Hz}) ;{ }^{13} \mathrm{C} \mathrm{NMR}\left(\mathrm{CDCl}_{3}\right): \delta 154.7,150.1,133.7,130.5,129.3,127.8,127.6$, 127.3, 126.8, 126.1, 125.3, 121.9, 61.8, 31.7, 28.7, 23.0, 13.7; IR $\left(\mathrm{cm}^{-1}\right): 1615(\mathrm{~m}), 1590$ (m), $1519(\mathrm{~m}), 146(\mathrm{~m}) \mathrm{cm}^{-1}$; MS: (m/e) $315\left(\mathrm{M}^{+}, 70\right), 272\left(\mathrm{M}^{+}-\mathrm{C}_{3} \mathrm{H}_{7}, 100\right), 229(29)$, 185 (19), 316 (21); HRMS: Calcd. For $\mathrm{C}_{22} \mathrm{H}_{21} \mathrm{NO}$ is 315.16232, found 315.16012.

\section{Coupling of Carbene Complex 6b with 2-hex-1-ynyl-benzaldehyde (2a) (Entry H):} General Procedure 4 was followed using carbene complex $6 \mathbf{b}(0.385 \mathrm{~g}, 1.10 \mathrm{mmol})$ and 2-hex-1-ynyl-benzaldehyde (2a) $(0.186 \mathrm{~g}, 1.0 \mathrm{mmol})$. The crude product was purified using flash chromatography on silica gel with hexane-ethyl acetate (9:1) as eluent to yield product 13h $(0.130 \mathrm{~g}, 40 \%) .{ }^{1} \mathrm{H}$ NMR $\left(\mathrm{CDCl}_{3}\right): \delta 9.44(\mathrm{~m}, 1 \mathrm{H}), 9.38(\mathrm{~s}, 1 \mathrm{H}), 8.79(\mathrm{~d}, 1$ $\mathrm{H}, \mathrm{J}=8.4 \mathrm{~Hz}$ ), 8.03-8.20 (m, $2 \mathrm{H}), 7.60-7.84$ (m, $4 \mathrm{H}), 3.53$ (t, $2 \mathrm{H}, \mathrm{J}=7.8 \mathrm{~Hz}), 3.19$ (s, 6 $\mathrm{H}), 1.55-1.75(\mathrm{~m}, 2 \mathrm{H}), 1.25-1.50(\mathrm{~m}, 2 \mathrm{H}), 0.91(\mathrm{t}, 3 \mathrm{H}, \mathrm{J}=7.0 \mathrm{~Hz}) ;{ }^{13} \mathrm{C} \mathrm{NMR}\left(\mathrm{CDCl}_{3}\right)$ : $\delta 151.0,147.9,141.2,135.1,133.6,132.5,132.3,129.4,128.6,127.7,127.1,126.4$, 126.3, 125.9, 125.7, 124.3, 122.6, 44.3 (2C), 32.1 (2C), 23.1, 13.9; IR (neat): 2915, 1940, 1615, $1379 \mathrm{~cm}^{-1}$; MS: (m/e) $330\left(\mathrm{M}^{+}+2,4\right), 329\left(\mathrm{M}^{+}+1,26\right), 328\left(\mathrm{M}^{+}, 86\right), 313\left(\mathrm{M}^{+}-\mathrm{CH}_{3}\right.$, 
8), 286 (22), 285 (100), 271 (85), 269 (53), 254 (10), 241 (16), 215 (6), 142 (13); HRMS: Calcd. For $\mathrm{C}_{23} \mathrm{H}_{24} \mathrm{~N}_{2}$ is 328.193949 , found 328.193884 .

Coupling of Carbene Complex 6b with (2-hex-1-ynyl-phenyl)-phenylmethanone (2c) (Entry I): General Procedure 4 was followed using carbene complex $6 \mathbf{b}(0.088 \mathrm{~g}, 0.25$ $\mathrm{mmol})$ and (2-hex-1-ynyl-phenyl)-phenyl methanone (2c) $(0.055 \mathrm{~g}, 0.21 \mathrm{mmol})$. The crude product was purified using flash chromatography on silica gel with hexane-ethyl acetate (3:1) as eluent to yield product $13 \mathbf{i}(0.051 \mathrm{~g}, 60 \%)$. ${ }^{1} \mathrm{H}$ NMR $\left(\mathrm{CDCl}_{3}\right): \delta 9.50(\mathrm{~m}$, $1 \mathrm{H}), 8.82(\mathrm{~d}, 1 \mathrm{H}, \mathrm{J}=8.0 \mathrm{~Hz}), 8.29(\mathrm{~d}, 1 \mathrm{H}, \mathrm{J}=8.0 \mathrm{~Hz}), 8.16(\mathrm{~m}, 1 \mathrm{H}), 8.02(\mathrm{~d}, 1 \mathrm{H}, \mathrm{J}=$ $8.0 \mathrm{~Hz}), 7.50-7.85(\mathrm{~m}, 8 \mathrm{H}), 3.56(\mathrm{t}, 2 \mathrm{H}, \mathrm{J}=7.5 \mathrm{~Hz}), 3.23(\mathrm{~s}, 6 \mathrm{H}), 1.55-1.80(\mathrm{~m}, 2 \mathrm{H})$, $1.25-1.50(\mathrm{~m}, 2 \mathrm{H}), 0.93(\mathrm{t}, 3 \mathrm{H}, \mathrm{J}=7.3 \mathrm{~Hz}) ;{ }^{13} \mathrm{C} \mathrm{NMR}\left(\mathrm{CDCl}_{3}\right): \delta 157.9,148.0,140.2$, 140.0, 134.8, 133.9, 132.6, 132.4, 130.4 (2C), 128.6, 128.5, 128.2 (3C), 127.0, 126.7, 126.0, 125.9, 125.8, 125.7, 124.3, 122.1, 44.4 (2C), 32.4, 32.2, 23.2, 13.9; IR (neat): 2957, 1940, 1574, $1377 \mathrm{~cm}^{-1}$; MS: (m/e) $406\left(\mathrm{M}^{+}+2,3\right), 405\left(\mathrm{M}^{+}+1,24\right), 404\left(\mathrm{M}^{+}, 80\right)$, 389 (M+ - Me, 7), 361 (94), 347 (100), 331 (18), 316 (9), 172 (21); HRMS: Calcd. For $\mathrm{C}_{29} \mathrm{H}_{28} \mathrm{~N}_{2}$ is 404.225249 , found 404.226090 .

Computational Details. The following series of steps were utilized to compute the reaction energies. Each of the species in Scheme 4 was drawn in CHEM 3D and then minimized using MM2. A Gaussian input file was constructed from the MM2 energyminimized structure. The structure was again minimized using Gaussian $98 \mathrm{~W}^{7}$ at the B3LYP level using the 6-31 $\mathrm{G}^{*}$ basis set, followed by a frequency calculation also at the B3LYP level using the $6-31 \mathrm{G}^{*}$ basis set The frequency calculation was conducted using Gaussian $98 \mathrm{~W}$ at the B3LYP level using the $6-31 \mathrm{G}$ basis set. The total thermal energy and entropy were obtained for each species at $298 \mathrm{~K} ; \Delta \mathrm{H}$ and $\Delta \mathrm{S}$ were obtained through addition/subtraction of appropriate numbers based on the interconversions of interest. Free energy was calculated from the equation $\Delta \mathrm{G}=\Delta \mathrm{H}-\mathrm{T} \Delta \mathrm{S}$. Values obtained are listed below.

\footnotetext{
${ }^{7}$ Frisch, M.J.; Trucks, G.W.; Schlegel, H.B.; Scuseria, G.E.; Robb, M.A.; Cheeseman, J.R.; Zakrzewski, V.G.; Montgomery, J.A.; Stratmann, R.E.; Burant, J.C.; Dapprich, S.; Millam, J.M.; Daniels, A.D.; Kudin, K.N.; Strain, M.C.; Farkas, O.; Tomasi, J.; Barone, V.; Cossi, M.; Cammi, R.; Menucci, B.; Pomelli, C.; Adamo, C.; Clifford, S.; Ochterski, J.; Petersson, G.A.; Ayala, P.Y.; Cui, Q.; Morokuma, K.; Malick, D.K.; Rabuck, A.D.; Raghavachari, K.; Foresman, J.B.; Cioslowki, J.; Ortiz, J.V.; Stefanov, B.B.; Liu, A.; Liashenko, A.; Piskorz, P.; Komaromi, I.; Gomperts, R.; Martin, R.L.; Fox, D.J.; Keith, T.; Al-Laham, M.A.; Peng, C.Y.; Nanayakkara, A.; Gonzalez, M.; Callacombe, M.; Gill, P.M.W.; Johnson, B.G.; Chen, W.; Wong, M.W.; Andres, J.L.; Head-Gordon, M.; Replogle, E.S.; Pople, J.A., Gaussian, Inc., Pittsburgh, PA, 1998.
} 


\begin{tabular}{|c|c|c|}
\hline SPECIES & THERMAL ENERGY $(\mathrm{kcal} / \mathrm{mol})$ & ENTROPY (cal/molK) \\
\hline A & 95.896 & 87.646 \\
\hline B & 68.639 & 75.512 \\
\hline $\mathrm{C}$ & 167.372 & 113.045 \\
\hline $\mathrm{D}$ & 152.960 & 118.941 \\
\hline $\mathrm{E}$ & 153.760 & 100.289 \\
\hline $\mathrm{F}$ & 137.885 & 114.849 \\
\hline G & 138.400 & 99.236 \\
\hline $\mathrm{H}$ & 135.315 & 97.607 \\
\hline $\mathrm{CO}_{2}$ & 8.935 & 51.165 \\
\hline $\mathrm{Cr}(\mathrm{CO})_{6}$ & 40.544 & 120.736 \\
\hline \multirow[t]{2}{*}{$\mathrm{Cr}(\mathrm{CO})_{5}$} & 33.561 & 110.945 (Octahedral minus one CO) \\
\hline & 32.649 & 108.649 (Trigonal bipyramidal) \\
\hline
\end{tabular}

